\title{
Extending the Temporal Horizon of Autonomous Robots
}

\author{
Chrystopher L. Nehaniv, Naeem Assif Mirza, Kerstin Dautenhahn, and René \\ te Boekhorst
}

Adaptive Systems Research Group, University of Hertfordshire, College Lane, Hatfield Herts AL10 9AB, United Kingdom

C.L.Nehanivaherts.ac.uk

\begin{abstract}
We introduce mathematically rigorous metrics on agent experiences having various temporal horizons. Sensorimotor variables accessible to the agent are treated as information-theoretic sources modelled as random variables. The time series from the sensorimotor variables over a given temporal region for different behavioural contexts ground an agent-based view of the agent's own experiences, and the information-theoretic differences between sensorimotor experiences induce a metric space structure on the set of the agent's possible experiences. This could allow an autonomous mobile robot to locate and navigate between its sensorimotor experiences on a geometric landscape (an experiential metric space) whose points are its possible experiences of a given temporal scope and in which nearby points are similar experiences.
\end{abstract}

\section{Motivation and Overview}

In order to mathematically formalize and exploit the notion of temporally extended episodes of experience for autonomous robots, we develop real-time computable information-theoretic metrics on a robot's sensorimotor experiences having a given temporal horizon. These are illustrated with a particular robotic example, and next applications to ontogenetic robots are discussed.

\section{Experience Metrics: The Geometry of Experience}

\subsection{Information Sources as Random Variables}

Consider a sensor or effector that can take on various settings or values modeled as a random variable $\mathcal{X}$ changing with time, taking value $x(t) \in X$, where $X$ is the set of its possible values. For simplicity in this paper, we take time to be discrete 
(i.e. $t$ will denote a natural number) and allow $\mathcal{X}$ to take values in a finite set or "alphabet" $X=\left\{x_{1}, \ldots, x_{m}\right\}$ of possible values. (The approach clearly generalizes to continuous time and value sets with appropriate changes.)

\subsection{Entropy and Information Distance}

Entropy is the information-theoretic measure of uncertainty introduced by Claude Shannon [10] and its units are bits. The entropy $H(\mathcal{X})$ of a sensor or actuator $\mathcal{X}$ is then $H(\mathcal{X})=-\sum_{x \in X} p(x) \log _{2} p(x)$, where $p(x)$ gives the probability of value $x$ being taken. Conditional entropy $H(\mathcal{X} \mid \mathcal{Y})$ of a random variable $\mathcal{X}$ given $\mathcal{Y}$ is the amount of uncertainty that remains about the value $\mathcal{X}$ given that the value of $\mathcal{Y}$ is known.

$$
H(\mathcal{X} \mid \mathcal{Y})=-\sum_{x \in X} \sum_{y \in Y} p(x, y) \log _{2} \frac{p(x, y)}{p(y)}
$$

where $p(x, y)$ is given by the joint distribution of $\mathcal{X}$ and $\mathcal{Y} .{ }^{1}$ The information distance between $\mathcal{X}$ and $\mathcal{Y}$ is

$$
d(\mathcal{X}, \mathcal{Y})=H(\mathcal{X} \mid \mathcal{Y})+H(\mathcal{Y} \mid \mathcal{X})
$$

This satisfies the mathematical axioms for a metric:

1. $d(\mathcal{X}, \mathcal{Y})=0$ if and only if $\mathcal{X}$ and $\mathcal{Y}$ are equivalent. ${ }^{2}$

2. $d(\mathcal{X}, \mathcal{Y})=d(\mathcal{Y}, \mathcal{X})$ (symmetry)

3. $d(\mathcal{X}, \mathcal{Y})+d(\mathcal{Y}, \mathcal{Z}) \geq d(\mathcal{X}, \mathcal{Z})$ (triangle inequality).

The satisfaction of these axioms is shown by Crutchfield [1]. Thus $d$ defines a geometric structure on any space of jointly distributed information sources.

\subsection{Sensorimotor Variables with Time Horizons}

For a particular agent, in a particular environment, consider a sensorimotor variable $\mathcal{X}$. Its distribution will be affected by the agent-environment interaction. In the context of a particular environment and beginning from a particular moment in time $t_{0}$ until a later moment $t_{0}+h(h>0)$, we regard the sequence of values $x\left(t_{0}\right), x\left(t_{0}+1\right), \ldots, x\left(t_{0}+h-1\right)$ taken by an information source $\mathcal{X}$ as time-series data from a new random variable $\mathcal{X}_{t_{0}, h}$, the sensorimotor variable with temporal horizon $h$ for sensor (or actuator) $\mathcal{X}$ starting at time $t_{0}$, depending on situated experience.

\footnotetext{
${ }^{1}$ We assume approximate local stationarity of the joint distribution of random variables representing the sensorimotor variables over a temporal window and that this can be estimated closely enough by sampling the sensorimotor variables.

${ }^{2}$ For information sources, "equivalence" refers to re-coding equivalence. That is, the values of $\mathcal{X}$ are a function of those of $\mathcal{Y}$ and vice versa. See [1].
} 


\subsection{Information Distance between Time-Shifted Sensorimotor Variables}

A particular robot engages in various behaviours and interactions in a particular environment, and we consider two of its sensorimotor variables $\mathcal{X}$ and $\mathcal{Y}$. (Possibly $\mathcal{X}=\mathcal{Y}$.) Consider the values taken by $\mathcal{X}$ beginning at time $t_{0}$ and those of $\mathcal{Y}$ beginning at time $t_{1}$. (Possibly $t_{0}=t_{1}$.) Consider the two-component random variable $\mathcal{X}_{t_{0}, h} \times \mathcal{Y}_{t_{1}, h}$ with horizon $h$, whose distribution is estimated from the values $\left(x\left(t_{0}+i\right), y\left(t_{1}+i\right)\right) \in X \times Y$. The first component here comes from $\mathcal{X}$, starting from time $t_{0}$, and second component comes from $\mathcal{Y}$ with a temporal shift of $t_{1}-t_{0}$ units, starting from time $t_{1}$. We can also estimate the probability joint time-shifted distribution and the information distance $d\left(\mathcal{X}_{t_{0}, h}, \mathcal{Y}_{t_{1}, h}\right)$ between $\mathcal{X}$ during the first temporal region and $\mathcal{Y}$ during the second temporal region by measuring the frequencies of occurrence of values $\left(x_{t_{0}+i}, y_{t_{1}+i}\right)$ as $i$ runs from 0 to $h-1$.

Clearly there are issues related to the size of the temporal horizon $h$ and also the number of values $\mathcal{X}$ and $\mathcal{Y}$ may take that affect the accuracy of these estimates. Also in practice, independent samples of time shifted sensorimotor variables are not available. ${ }^{3}$

\subsection{Experience Metric}

Consider the set of all sensorimotor variables available to an agent. Suppose there are $N$ such, $\mathcal{X}^{1}, \ldots, \mathcal{X}^{N}$. Let $E(t, h)=\left(\mathcal{X}_{t, h}^{1}, \ldots, \mathcal{X}_{t, h}^{N}\right)$ be the (ordered) set of these variables considered over a temporal window of size $h$ starting at $t$. We call $E(t, h)$ the agent's experience from time $t$ having temporal horizon $h$.

Let $E=E(t, h)$ and $E^{\prime}=E\left(t^{\prime}, h\right)$ be experiences of an agent from time $t$ and $t^{\prime}$, respectively, both with horizon size $h$. Define a metric on experiences of temporal horizon $h$ as

$$
D\left(E, E^{\prime}\right)=\sum_{k=1}^{N} d\left(\mathcal{X}_{t, h}^{k}, \mathcal{X}_{t^{\prime}, h}^{k}\right)
$$

where $d$ is the information distance.

Theorem $1 D$ is a metric on the set of experiences of an agent having a fixed temporal horizon $h$.

Proof: That the metric axioms holds for $D$ follows from the fact that they hold componentwise, since $d$ is a metric.

As a corollary, we note that $\bar{D}=\frac{1}{N} D$, the average experience distance per sensorimotor variable, is also a metric on the set of experiences of an agent having a fixed

\footnotetext{
${ }^{3}$ Previous work on sensory reconstruction and sensorimotor learning by Olsson et al. [8, 7] can be considered as using as large as possible horizon (starting $t_{0}=0$ and $h$ the number of observations made). Sometimes also temporal shifts by a small amount $t_{1}-t_{0}$ to study temporal correlations in information (such as occur, e.g. in optical or tactile flow) [9].
} 
temporal horizon $h$. The units of $D$ are bits and those of $\bar{D}$ are bits per sensorimotor variable. Thus $D$ provides a geometric structure on the set of experiences of an agent. $^{4}$

\section{Autonomous Robot Experiments}

\subsection{Experimental Set-up}

Sensorimotor variable data for a SONY AIBO robot programmed with behaviourbased techniques to explore a $2 \mathrm{~m} \times 2 \mathrm{~m}$ environment were gathered previously to study information distance characterizations of sensorimotor interactions [3]. These data are reanalyzed here using the tools of the experience metric introduced above for temporal horizon of size 40 with resolution of five values for each sensorimotor variable $\mathcal{X}$ (i.e. $|X|=5$ ), where time is measured in units of approx. $100 \mathrm{msec}$. More details of programming and data-gathering, sensitivity to parameters, and examination of other aspects of the temporal experience metric are described in [4]. The seventy-seven sensorimotor variables used can be partitioned into two classes (read-only) variables (41 "sensors") and (read-write) variables (36 "motors") - see Table 1.

Table 1. AIBO Telemetry Collected

\begin{tabular}{|l|r|l|r|}
\hline Sensors & $\#$ & Motors & $\#$ \\
\hline IR-Distance & 1 & Leg Joint Positions & 12 \\
Accelerometers & 3 & Head Joint Positions & 4 \\
Temperature/Battery & 2 & Tail Joint Positions & 2 \\
Buttons & 8 & Motor Force / Duties & 18 \\
Visual & 27 & & \\
\hline Total Sensors & 41 & Total Motors & 36 \\
\hline
\end{tabular}

Data from sensorimotor variables was sampled at approximately 10 times per sec. Figure 1 shows the trajectory of the robot in the arena lasting 90.3 seconds. For this data set, the experience metric was used to examine the similarity of 17 sensorimotor experiences ending at the waypoints shown.

\subsection{Results}

Figure 2 shows a local neighborhood in the experiential metric space for experiences in this data set: Centering at walk 5 (see caption) the closest experiences in the data set are the temporal region walk 9 leading up to waypoint 9 , lying just beyond the

\footnotetext{
${ }^{4}$ More detailed proofs and other metrics on sensorimotor experience (in the sense of this paper) are given in [5].
} 


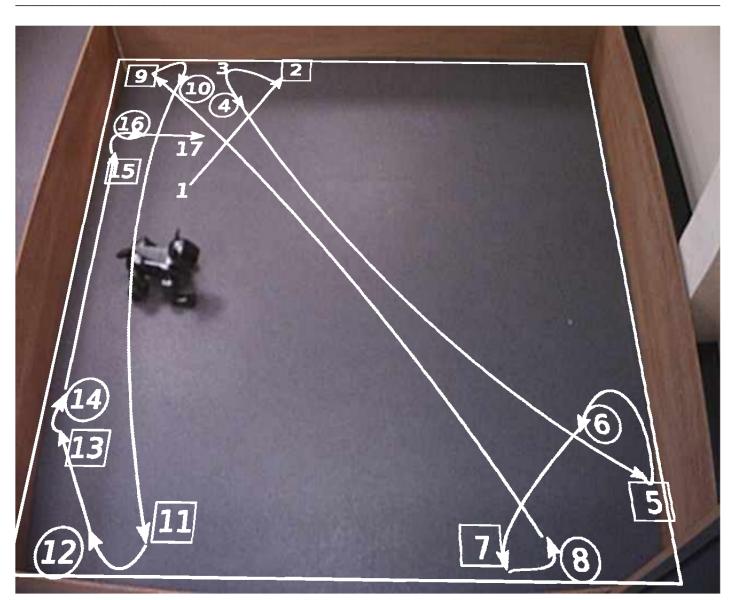

Fig. 1. Trajectory of the autonomously wandering AIBO robot in $2 \mathrm{~m} \times 2 \mathrm{~m}$ arena. Numbered waypoints mark ends of temporal windows considered. For each, behaviour in the temporal horizon $(h=40)$ were characterized by external observers as either walk (circles) or turn (squares); waypoint 1 involved the robot's start-up.

ball of radius 40 bits centered at walk 5 (w5), followed by walk11 (w11), which lies within the ball of radius 60 bits. Turns (prefixed $t$ ) and start up s 1 lie further away.

Figure 3 shows a dendrogram resulting from complete linkage clustering of the experiences using the experience metric $D$. Generally, clusters consist of experiences externally characterized by human observers as either walking or turning, but the robot-centered metric splits off some subclusters and gives finer distinctions.

\section{Discussion and Conclusion}

We have shown how an autonomous robot can apply a metric (computed in realtime) to assess the similarity of its experiences in a geometric space consisting of its possible experiences over a given size temporal horizon, and that these tend to agree with external observer notions of similarity. The next steps are to apply this experience metric to allow the robot to help it to re-engage in known experience, to predict and explore experiences near the boundaries of its previously mastered behaviours and experiences (cf. [12, 11, 2, 6]).

We remark that the systematic comparison of experience at different temporal horizons is possible using restriction mappings and the experience metric at the shorter scales [5]. Morever, as development progresses, fine resolution on sensorimotor variables could be mapped naturally onto an initial coarse resolution of sensorimotor variables to scaffold ontogeny. 


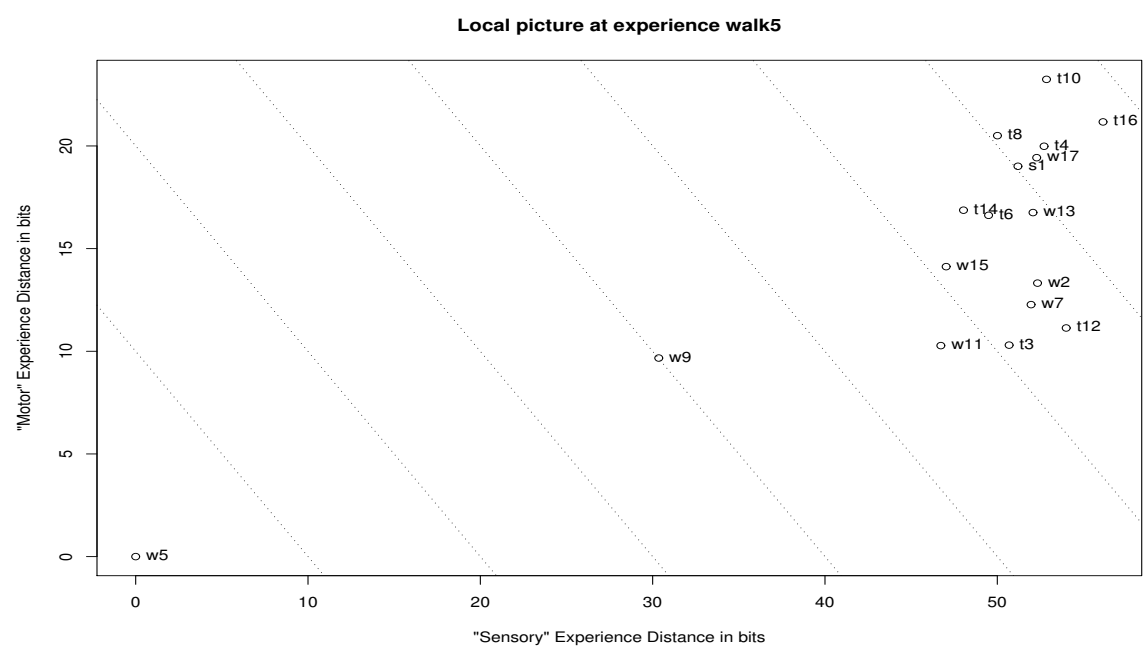

Fig. 2. Local neighborhood in the experience metric space. For the sensorimotor experience walk 5 (w5) ending at waypoint 5 with a temporal region with horizon of size 40 and resolution (bin size) of 5 values per sensorimotor variable, experiential distances to other experiences in the data are visualized. Points are experiences human-characterized either as walk, turn or start-up denoted by first letter $w$, $t$, or $s$ followed by waypoint number (see Figure 1). Horizontal axis (resp. vertical axis) shows contribution in bits to experience distance from "sensory" read-only variables (resp. "motor" read-write variables). Experience distance to the experience at the origin is the sum of these two coordinates. Dotted lines show the boundary of the balls with radii 10-80 around experience walk 5. Caveat: Distances between other experiences depicted are generally much larger than they appear in the local picture.

Acknowledgments. The work described in this paper was in part conducted within the EU Integrated Project RobotCub ("Robotic Open-architecture Technology for Cognition, Understanding, a nd Behaviours") and was funded in part by the European Commission through the E5 Unit (Cognition) of FP6-IST under Contract FP6-004370.

\section{References}

1. CRUtchfield, J. P. Information and its metric. In Nonlinear Structures in Physical Systems - Pattern Formation, Chaos and Waves, L. Lam and H. Morris, Eds. SpringerVerlag, 1990, pp. 119-130.

2. Dautenhahn, K., And Christaller, T. Remembering, rehearsal and empathy towards a social and embodied cognitive psychology for artifacts. In Two Sciences of the Mind. Readings in cognitive science and consciousness, S. O'Nuallain and P. McKevitt, Eds. John Benjamins Publ., 1996, pp. 257-282.

3. Mirza, N. A., Nehaniv, C. L., Dautenhahn, K., And te Boekhorst, R. Using sensory-motor phase-plots to characterise robot-environment interactions. In Proc. 6th 


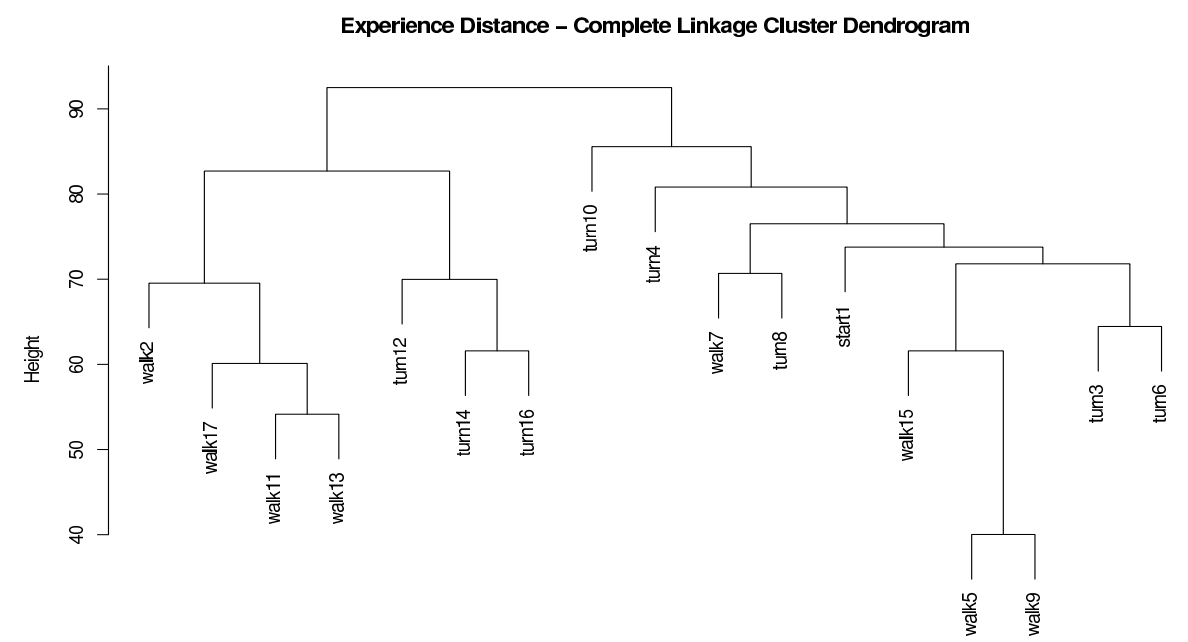

Fig. 3. Dendrogram for experiences clustered using experience metric. Experiences of temporal horizon $4.0 \mathrm{sec} .(40 \times 100 \mathrm{msec}$. $)$ ending at waypoints in the autonomous robot exploratory behaviour of Figure 1. (Complete linkage clustering method was used; Ward method dendrogram results are similar.)

IEEE International Symposium on Computational Intelligence in Robotics and Automation (CIRA'05) (2005), pp. 581-586.

4. Mirza, N. A., Nehaniv, C. L., Dautenhahn, K., And te Boekhorst, R. Using temporal information distance to locate sensorimotor experience in a metric space. In Proc. IEEE Congress on Evolutionary Computation (2005).

5. Nehaniv, C. L. Sensorimotor experience and its metrics: Informational geometry and the temporal horizon. In Proc. IEEE Congress on Evolutionary Computation (2005).

6. Nehaniv, C. L., Polani, D., Dautenhahn, K., te Boekhorst, R., And CAñAmero, L. Meaningful information, sensor evolution, and the temporal horizon of embodied organisms. In Artificial Life VIII. MIT Press, 2002, pp. 345-349.

7. Olsson, L., Nehaniv, C. L., And Polani, D. The effects on visual information in a robot in environments with oriented contours. In Fourth International Workshop on Epigenetic Robotics (EpiRob'04) (2004), pp. 83-88.

8. Olsson, L., Nehaniv, C. L., AND Polani, D. Sensory channel grouping and structure from uninterpreted sensor data. In IEEE NASA/DoD Conference on Evolvable Hardware (2004), pp. 153-160.

9. Olsson, L., Nehaniv, C. L., And Polani, D. Discovering motion flow by temporalinformational correlations in sensors. In Proc. Fifth International Workshop on Epigenetic Robotics (EpiRob'05) (2005).

10. Shannon, C. E. A mathematical theory of communication. Bell Systems Technical Journal 27 (1948), 379-423 and 623-656.

11. VArela, F. J., Thompson, E., AND Rosch, E. The Embodied Mind: Cognitive science and human experience. MIT Press, 1991.

12. VyGOTSKY, L. S. Mind and Society: The development of higher mental processes. Harvard University Press, 1978. 